\title{
The system modelling and circuit implementation from time-frequency domain signal specifications
}

\author{
F. Acar Savac1 ${ }^{\mathrm{a}}$, Nalan Özkurt ${ }^{\mathrm{b}, *}$ \\ ${ }^{a}$ Department of Electrical-Electronics Engineering, Izmir Institute of Technology, Turkey \\ ${ }^{\mathrm{b}}$ Department of Electrical-Electronics Engineering, Dokuz Eylul University, Turkey
}

Received 8 August 2005; accepted 28 November 2006

\begin{abstract}
The system modelling and the circuit implementation of the nonlinear circuits using the wavelet domain techniques has been accomplished in this study. When the time-frequency domain specifications have been given as the wavelet ridges, the signal with the given ridges has been synthesized. Then, the dynamical wavelet network has been trained for the synthesized signal. The circuit of the wavelet network has been designed and simulated.
\end{abstract}

(c) 2007 Elsevier GmbH. All rights reserved.

Keywords: Wavelets; Circuit realization; Function approximation

\section{Introduction}

Although the dynamic behavior of linear networks is fully determined, the behavior of nonlinear dynamic circuits can be extremely complex and unpredictable. Furthermore, there are no certain procedures especially for the synthesis of nonlinear circuits, instead there are specific solutions for several applications. Since the time-domain methods and frequency-domain methods alone do not provide adequate information about the dynamics of such signals, there have been several studies on the analysis of the nonlinear dynamical systems with time-frequency domain methods [1-6]. Also, there have been studies on the reconstruction of the attractors of the chaotic systems using the Wigner-Ville distribution [7] and the wavelet transform [8]. The wavelet network [9] which combines feedforward neural networks and wavelet decompositions, have attracted many

\footnotetext{
* Corresponding author. Tel.: +902324531008.

E-mail addresses: acarsavaci@iyte.edu.tr (F.A. Savac1), nalan.ozkurt@eee.deu.edu.tr (N. Özkurt).
}

researchers since the wavelet analysis has been successfully applied for analyzing signals both in space and frequency domain with different resolution levels [10-12]. Additionally, the wavelet network is powered with the statistical techniques in [13].

In this study, a circuit design procedure for the nonlinear circuits with desired time-frequency domain behavior using wavelet domain methods has been proposed [14]. The system includes four main blocks: signals synthesis, system modelling, circuit synthesis and verification which are shown in Fig. 1. The signal synthesis block synthesizes the signal with desired time-frequency domain properties using wavelet ridges method [15]. The system modelling block determines the parameters of the dynamical wavelet network using the time-series synthesized by the first block. In the circuit synthesis block, the wavelet network circuit has been realized with the wavelon circuit proposed in [16-18]. In the verification phase, the wavelet ridges of the output of the wavelet network have been calculated by singular value decomposition-based ridge determination method proposed in [19]. 


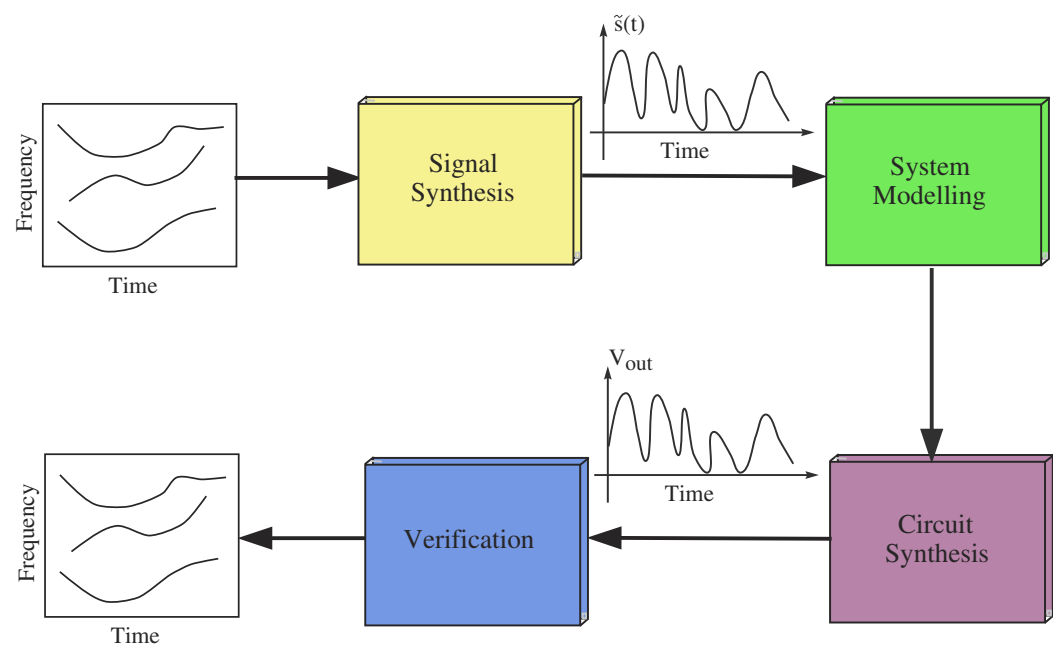

Fig. 1. The block diagram of the system.

\section{Signal synthesis}

The signal synthesis block synthesizes the signal with given time-frequency domain specifications as follows:

$E(t, f)=\left\{\begin{array}{ll}E_{1}(t, f) & t_{0} \leqslant t<t_{1} \quad \text { and } f_{0} \leqslant f<f_{1} \\ \vdots & \\ E_{n}(t, f) & t_{n-1} \leqslant t<t_{n} \\ 0 & \text { otherwise }\end{array}\right.$ and $f_{n-1} \leqslant f<f_{n}$,

where $E(t, f)$ represents the energy distribution in the time-frequency plane.

In this section, before explaining the signal synthesis using wavelet ridges, the necessary definitions and concepts about the wavelet transform and wavelet ridges will be given.

\subsection{Wavelet transform}

In continuous wavelet transform (CWT), the signal is projected on a family of zero-mean functions called wavelets, deduced from a mother wavelet by translations and dilations

$W_{s}(a, b ; \Psi) \triangleq \int_{-\infty}^{\infty} s(t) \Psi_{a, b}^{*}(t) \mathrm{d} t$,

where $a$ and $b$ are the dilation (scale) and translation coefficients, respectively; the scaled and translated wavelet is obtained as

$\Psi_{a, b}(t) \triangleq \frac{1}{a} \Psi\left(\frac{t-b}{a}\right), \quad a \in \mathbb{R}^{+}, \quad b \in \mathbb{R}$,

where $\Psi(\cdot)$ is the mother wavelet and $*$ denotes the complex conjugate.

The local time-frequency energy density which is called scalogram $P_{S}(a, b ; \Psi)$ has been defined in the wavelet domain as in [20]

$P_{S}(a, b ; \Psi) \triangleq\left|W_{s}(a, b ; \Psi)\right|^{2}$.
The continuous wavelet transform conserves the total energy of the signal $E_{\mathrm{T}}$ according to the Plancherel's formula as in [21]

$$
\begin{aligned}
E_{\mathrm{T}} & \triangleq\|s\|_{2}^{2}=\int_{-\infty}^{\infty}|s(t)|^{2} \mathrm{~d} t \\
& =c_{\psi}^{-1} \int_{-\infty}^{\infty} \int_{0}^{\infty}\left|W_{s}(a, b ; \Psi)\right|^{2} \frac{\mathrm{d} a \mathrm{~d} b}{a}
\end{aligned}
$$

where $c_{\psi}$ is the admissibility constant.

For the numerical computations, the discrete samples of the continuous wavelet transform have been considered and the scaled and translated wavelets have been defined at the dyadic grid as

$\Psi_{m, n}(t)=a_{0}^{-m / 2} \Psi\left(a_{0}^{-m} t-n b_{0}\right) \quad m, n \in \mathbb{Z}$,

where $a_{0}, b_{0} \in \mathbb{R}$ are the dilation and translation coefficients, respectively.

If the wavelets defined in Eq. (6) are chosen such as to constitute a Riesz basis for every $s(t) \in L^{2}(\mathbb{R})$, by considering only finite number of basis vectors for practical purposes, the total energy in Eq. (5) can be approximately given in [19] as

$\tilde{E}_{T} \triangleq \frac{k}{c_{\psi}} \sum_{m=1}^{M} \sum_{n=1}^{N} p_{m n}$

where $k \triangleq b_{0} \ln a_{0}$ and the entries of the scalogram matrix $P=\left[p_{m n}\right]_{M \times N}$ are defined as

$p_{m n} \triangleq\left|c_{m n}\right|^{2}$

which are the local time-frequency domain energy densities evaluated at the discrete dilations $a_{m}$ and translations $b_{n}$. 


\subsection{Wavelet ridges}

A way of giving the time-frequency domain specifications is to define the ridge of the time-frequency transform (wavelet or Gabor transforms) or the instantaneous frequency of the signal. The instantaneous frequency of a signal is simply defined as the derivative of the phase of the signal, and therefore the signals can be modelled by using a frequency-modulated signal fitted to the change of the main frequency.

The multi-component signal with the instantaneous amplitudes $A_{l}(t)$ and the instantaneous phases $\phi_{l}(t)$ can be described by

$s(t)=\sum_{l=1}^{L} A_{l}(t) \mathrm{e}^{\mathrm{j} \phi_{l}(t)}$,

where $L$ is the number of the components, then the wavelet transform can be written as

$W_{s}(a, b ; \Psi)=\frac{1}{2} \sum_{l=1}^{L} A_{l}(b) \mathrm{e}^{\mathrm{j} \phi_{l}(b)} \hat{\Psi}^{*}\left(a \phi_{l}^{\prime}(b)\right)+r(a, b)$

with $r(a, b) \sim O\left(\left|A_{l}^{\prime}\right|,\left|\phi_{l}^{\prime \prime}\right|\right)$ where the prime denotes the derivative $[21,22]$. Therefore, if the Fourier transform of the mother wavelet " $\hat{\Psi}(\omega)$ " is localized near a certain frequency $\omega=\omega_{0}$, the scalogram is localized around $L$ curves

$a^{l}=a^{l}(b)=\frac{\omega_{0}}{\phi_{l}^{\prime}(b)}, \quad l=1, \ldots, L$,

which are called ridges of the wavelet transform. The values of the scalogram along the ridge construct the transform skeleton or wavelet curve. Thus, the original signal $s(t)$ can be recovered using the skeleton of the transform.

\subsection{Signal synthesis using wavelet ridges}

The reconstruction of the signal from the wavelet ridges for mono-component and multi-component signals have been given in [22] and [23], respectively.

The real part of the signal $s(t)$ given in Eq. (9) can be constructed from the skeleton of the transform using the approximate formula Eq. (10) as

$s_{r}(b)=2 \operatorname{Re}\left\{\sum_{l=1}^{L} W_{s}\left(a^{l}(b), b ; \Psi\right)\right\}$

where $L$ is the number of the ridges. The reconstruction using the transform skeleton is a simple scheme and it produces good approximations. However, it requires the knowledge of the transform at each point of the ridge [22]. Therefore, each point along the ridge should be given for synthesis, or the gaps along the ridges should be completed using interpolation or curve-fitting techniques. The synthesis program

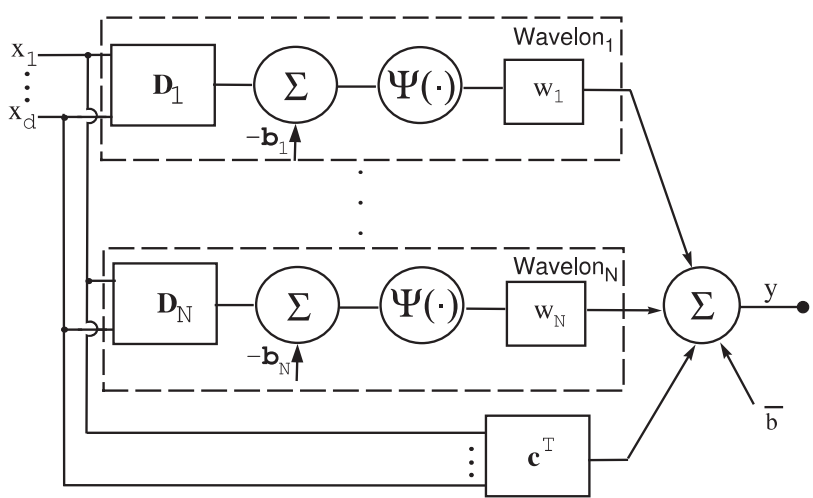

Fig. 2. The block diagram of the static wavelet network.

has been written in MATLAB [15]. The time-frequency domain specifications of the wavelet ridges of signals have been given interactively by the user. The monocomponent and multicomponent signals with sinusoidal, hyperbolic or linear ridges can be obtained. Also, the specific points of the ridges in time-frequency domain can be given by the user.

\section{System modelling}

Any finite energy multivariate function can be approximated by wavelets using the multiresolution approximation property of the wavelet decomposition. In order to approximate arbitrary nonlinear functions the wavelet network which combines feedforward neural networks and wavelet decompositions has been proposed in [9]. The identification of static and dynamical systems using wavelet network have attracted many researchers since the wavelet analysis has been successfully applied for analyzing signals both in time and frequency domain with different resolution levels [10-13].

When the input-output pairs measured from the system to be modelled is given as

$$
\begin{aligned}
& \left\{x\left(t_{k}\right), y\left(t_{k}\right) \mid y\left(t_{k}\right)=f\left(x\left(t_{k}\right)\right)+\varepsilon_{k}, \quad k=1,2, \ldots, K,\right. \\
& \left.\quad f(\cdot): \mathbb{R}^{d} \rightarrow \mathbb{R}\right\},
\end{aligned}
$$

where $\varepsilon_{k}$ is the measurement noise, the problem is to minimize the mean square error between the actual output and the output of the wavelet network

$\mathrm{MSE} \triangleq \frac{1}{2} E\left\{\left(y-f_{w}(x)\right)^{2}\right\}$,

where the output of the wavelet network is defined as

$f_{w}(x)=\sum_{i=1}^{N} w_{i} \Psi_{d}\left(D_{i}\left(x-b_{i}\right)\right)+c^{T} x+\bar{b}$,

where $N$ is the number of $d$-dimensional wavelons, $w_{i}$ is the wavelet coefficient for each $d$-dimensional wavelon, 


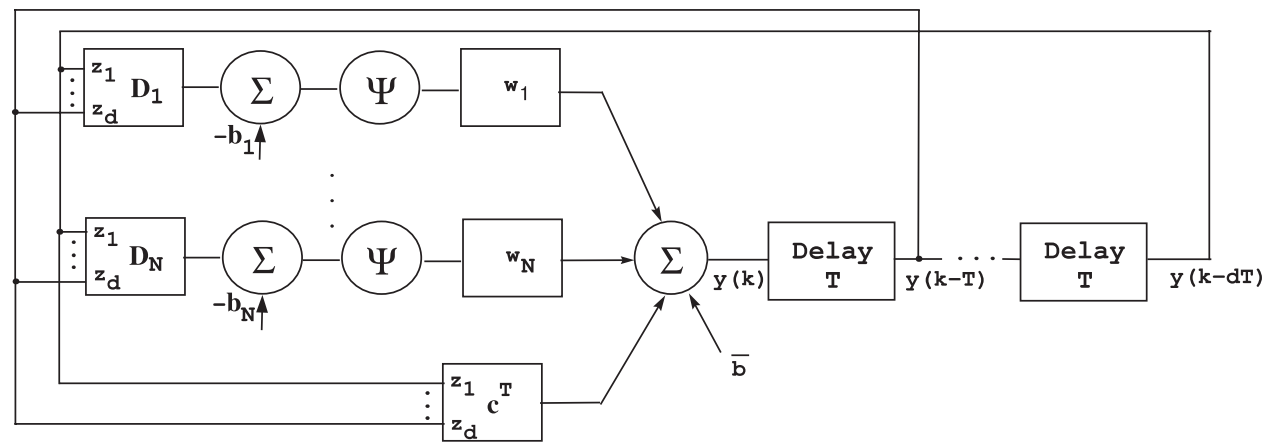

Fig. 3. The block diagram of the dynamical wavelet network.

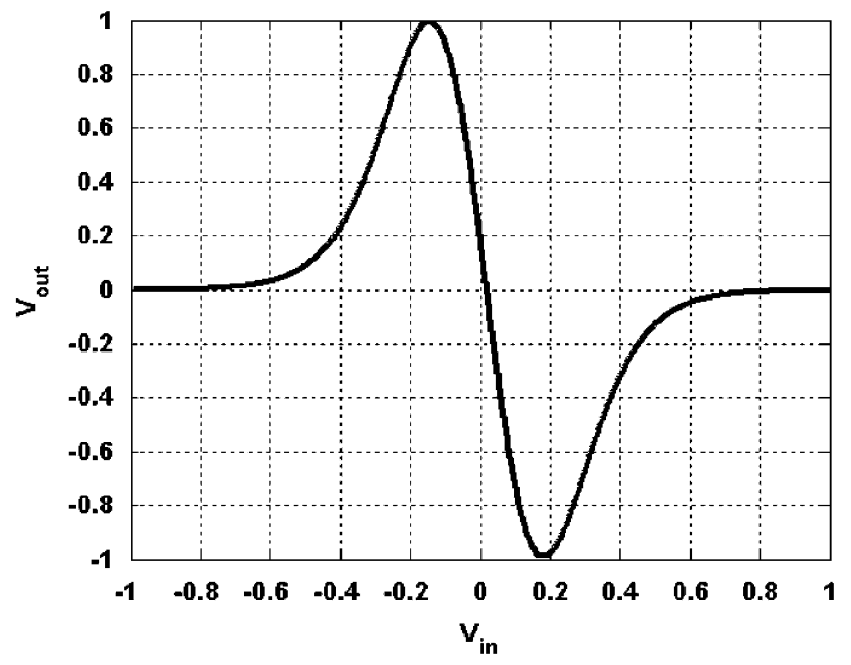

Fig. 4. The input-output voltage pair of wavelet circuit.

$D_{i}=\operatorname{diag}\left(d_{11}^{1}, \ldots, d_{d d}^{1}\right) \in \mathbb{R}^{d \times d}$ is the diagonal dilation matrix whose diagonal elements are $d_{j j}^{i}=1 / a_{i j}$ where $a_{i j}$ is the dilation coefficient, $\Psi_{d}(\cdot): \mathbb{R}^{d} \rightarrow \mathbb{R}$ is the mother wavelet function, and $b_{i} \in \mathbb{R}^{d}$ is the translation coefficient vector, $c \in \mathbb{R}^{d}$ represents the coefficient of the linear term and $\bar{b}$ is the bias term to approximate the functions with nonzero mean. The block diagram of the static wavelet network is shown in Fig. 2.

The optimum parameter set and the number of wavelons are to be determined for the construction of the wavelet network. The selection of suitable wavelons is implemented by the "Stepwise Selection by Orthogonalization" algorithm proposed in [13].

\subsection{Dynamical system modelling with wavelet network}

The wavelet networks have also been used in the identification of the dynamical systems or in the prediction of the future outputs of the systems. Assume that a dynamical

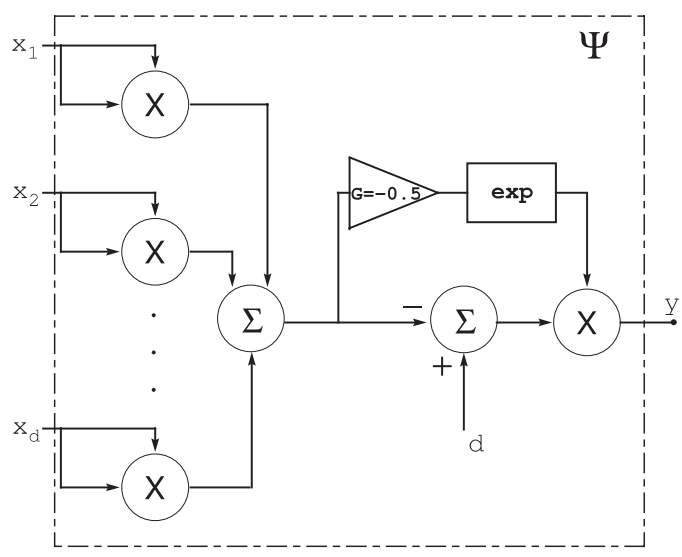

Fig. 5. The block diagram of Mexican hat mother wavelet circuit.

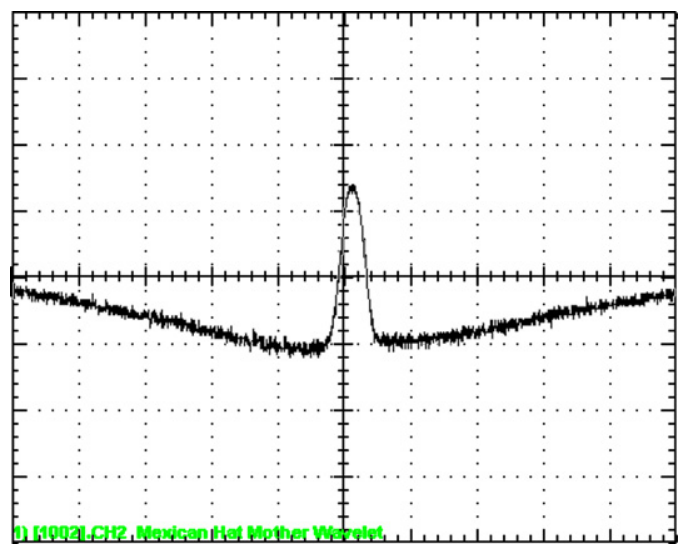

Fig. 6. The output of Mexican hat mother wavelet circuit.

system is defined as

$y(k)=F(x(k))+\varepsilon_{k}, \quad F(\cdot): \mathbb{R}^{d} \rightarrow \mathbb{R}$.

According to the Taken's time-delay embedding theorem [24], the multidimensional dynamical structure of the system can be retrieved from single scalar variable observed from the system. Let a vector $x_{s} \in \mathbb{R}^{d}$ is constructed from the 
(a)

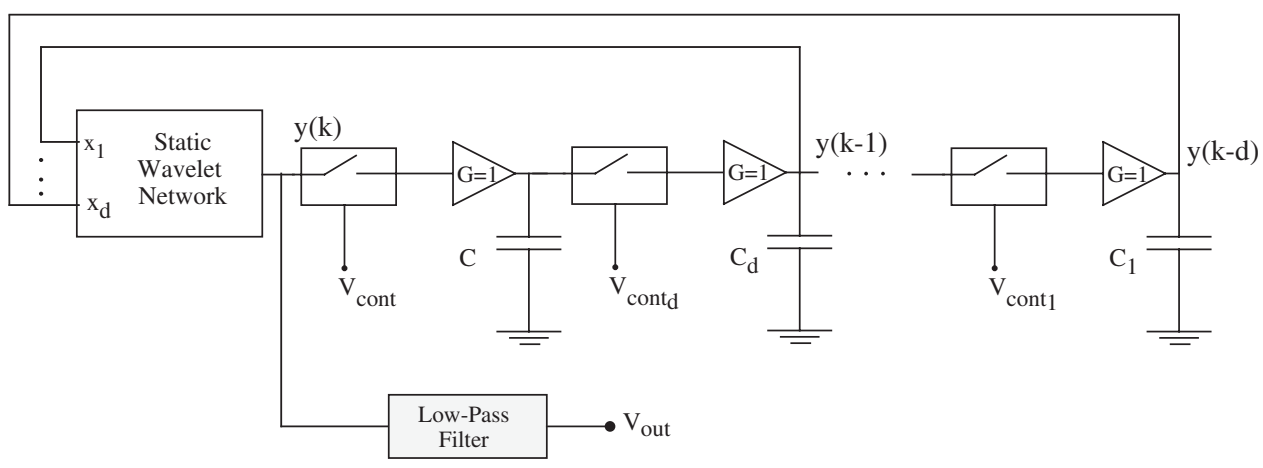

(b)

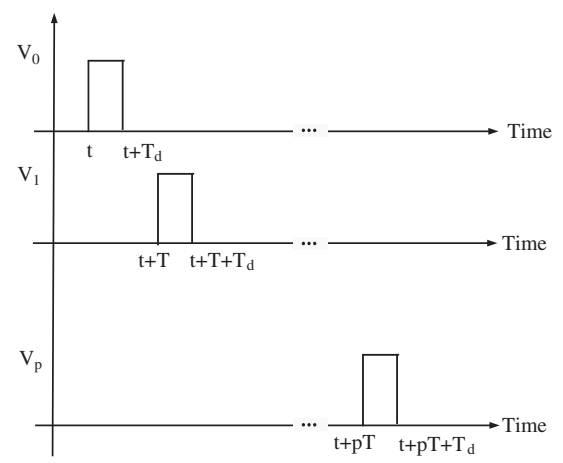

Fig. 7. (a) Dynamical wavelet network circuit and (b) the triggering pulses for voltage controlled switches.
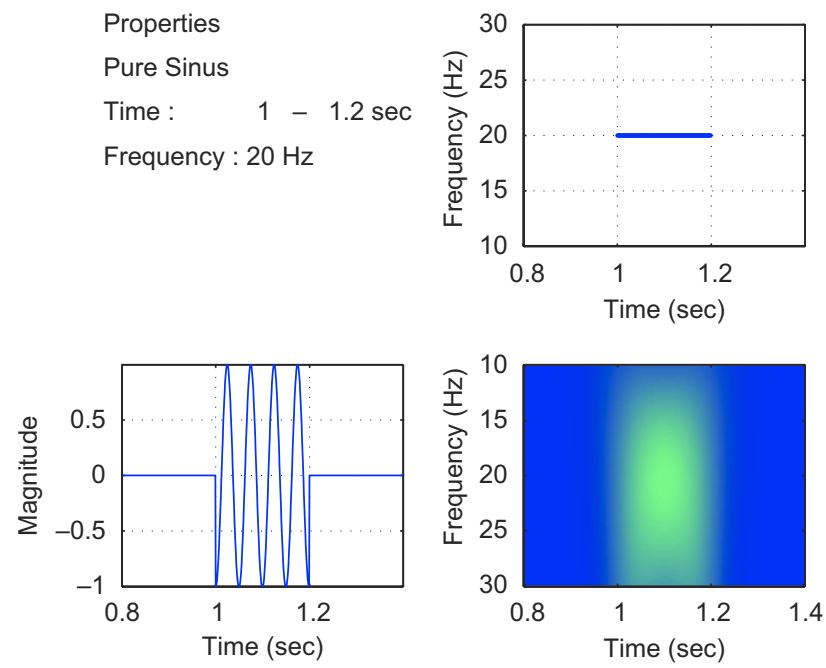

Fig. 8. The synthesized signal for Example 1 with wavelet ridges method.

observations as

$x_{s}(k)=[y(k-T) y(k-2 T) \ldots y(k-d T)]$,

where $d$ is the embedding dimension and $T$ is the embedding delay. The

$y(k)=F\left(x_{S}(k)\right)$ follows the dynamical evolution of the original system. Therefore, the next state of the system is predicted from the previous observations.

The system evolution function $F$ is approximated by some arbitrary set of basis functions for the modelling or identification of the nonlinear dynamical systems [10-12]. The purpose is to represent Eq. (18) with the suitable wavelet network. Since the observations can be expressed as a function of past measurements, the past values are used as inputs and the present values are used as output for the wavelet network to approximate the function $F(\cdot)$. The output of the wavelet network $F_{W}$ is

$$
\begin{aligned}
y(k) & =F_{w}\left(x_{s}(k)\right) \\
& =\sum_{i=1}^{N} w_{i} \Psi_{d}\left(D_{i} x_{s}(k)-b_{i}\right)+c^{T} x+\bar{f} .
\end{aligned}
$$

The block diagram of the dynamical wavelet network is shown in Fig. 3.

\section{Circuit synthesis}

The static and dynamical wavelet networks successfully model the nonlinear systems. The circuit implementation of the wavelet network with sigmoidal mother wavelet [16] and Mexican Hat mother wavelet [17] will be introduced in this section. 

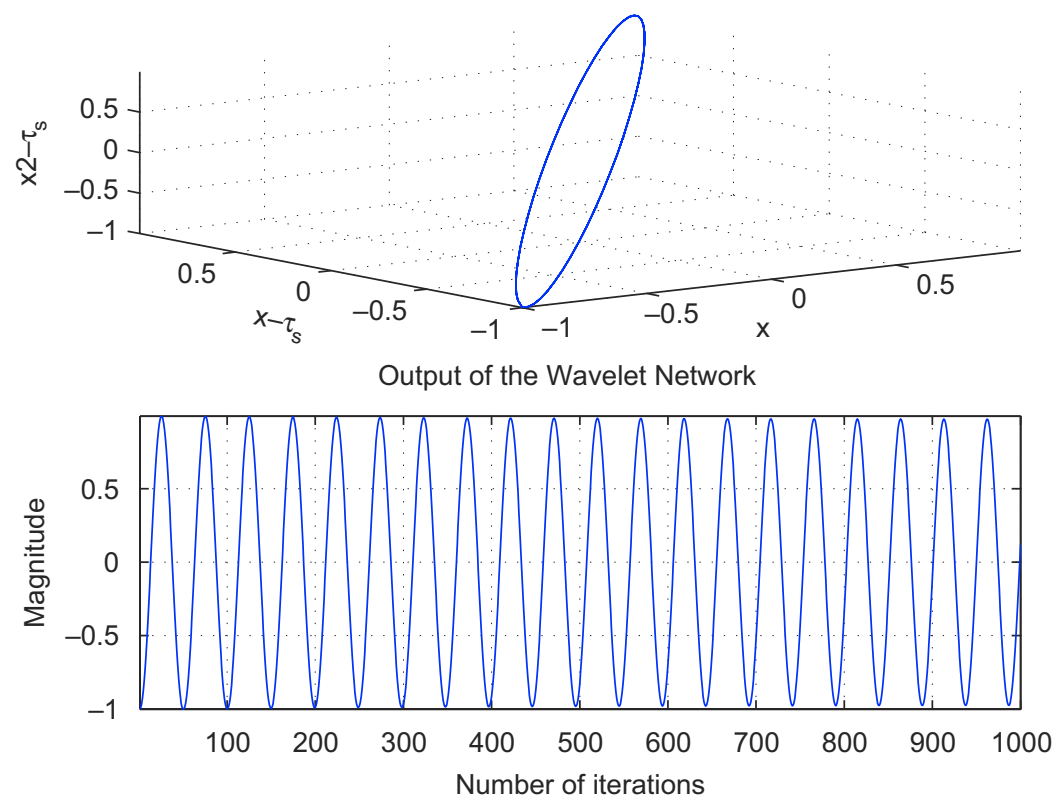

Fig. 9. The embedded monocomponent signal in phase space and the output of the wavelet network.

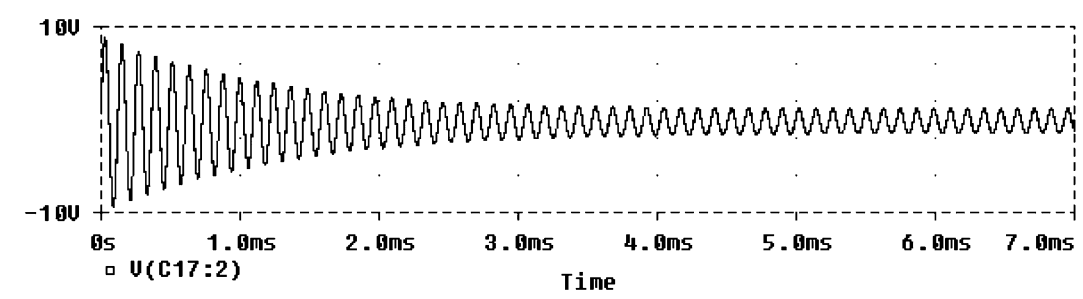

Fig. 10. The output of the circuit for monocomponent signal.

\subsection{The circuit implementation of sigmoidal mother wavelet}

The sigmoidal mother wavelet function, which has been proposed in [25], can be implemented as the sum of three translated hyperbolic tangent functions as

$\Psi(x)=\tanh (x-2)-2 \tanh (x)+\tanh (x+2)$.

The hyperbolic tangent function can be implemented by a dual-transistor pair [16]. The input-output voltage pair of the wavelet circuit in the PSpice is shown in Fig. 4.

The circuit implementations of the tensor product wavelets are obtained as the analog multiplication of the one-dimensional wavelets.

\subsection{The circuit implementation of Mexican hat mother wavelet}

The Mexican Hat mother wavelet is one of the commonly used real mother wavelet because of its good approximation ability. The mother wavelet defined as

$\Psi(x)=\left(d-\|x\|^{2}\right) \exp \left(-\frac{\|x\|^{2}}{2}\right) \Psi(\cdot): \mathbb{R}^{d} \rightarrow \mathbb{R}$,

where $\|x\|^{2}=x^{T} x$.

The block diagram of the $d$-dimensional Mexican Hat mother wavelet circuit is shown in Fig. 5. The adders and amplifiers are implemented with operational amplifiers and the four channel four quadrant analog multiplier MLT04 of Analog devices has been used for multiplication and the exponential function is implemented with an antilog amplifier [17].

The measured input-output voltage pair of the Mexican Hat mother wavelet circuit is shown in Fig. 6.

\subsection{The circuit implementation of dynamical wavelet network}

The wavelet network can be used for modelling the nonlinear dynamical systems and the block diagram of the 


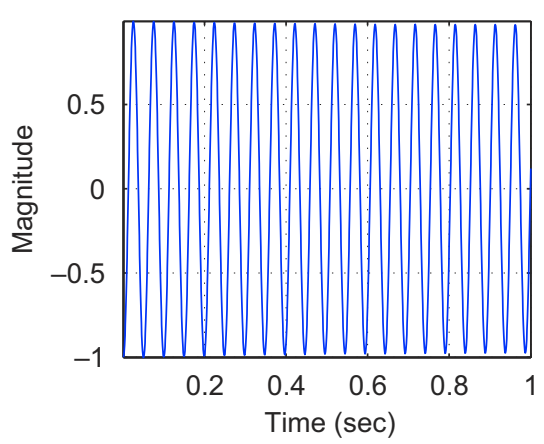

(a)

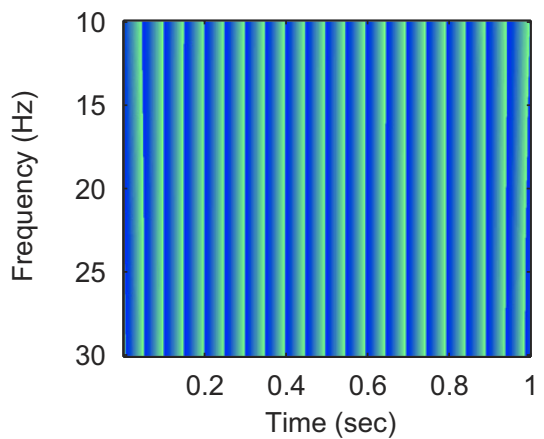

(c)

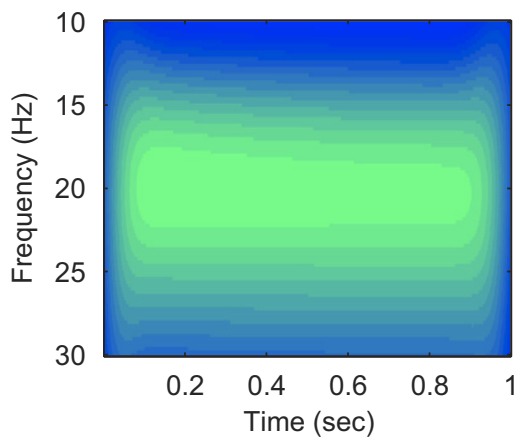

(b)

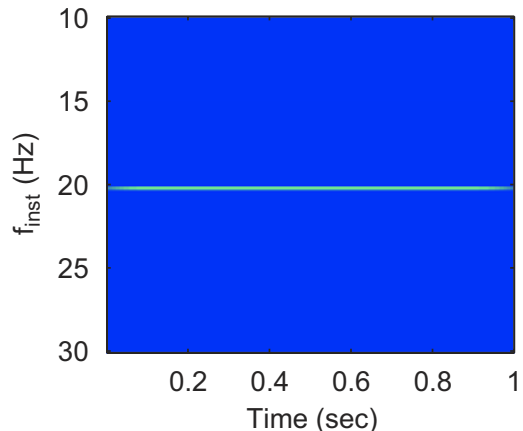

(d)

Fig. 11. (a) The time waveform, (b) the modulus, (c) the phase of the wavelet transform, and (d) the instantaneous frequency of the output of the wavelet network for monocomponent signal.

\begin{tabular}{|c|c|c|}
\hline \multicolumn{3}{|l|}{ Properties } \\
\hline Free Form & & \\
\hline Time : & 0.2 & $-0.8 \mathrm{sec}$ \\
\hline Frequency : & 4 & $-15 \mathrm{~Hz}$ \\
\hline
\end{tabular}

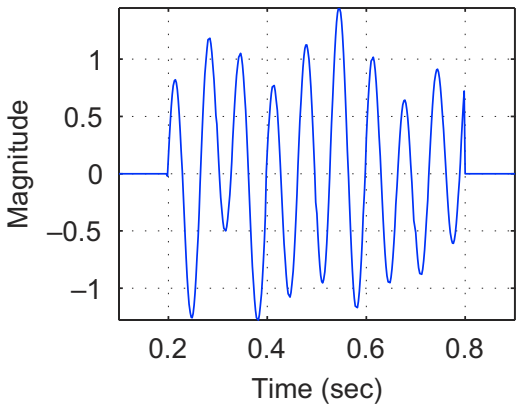

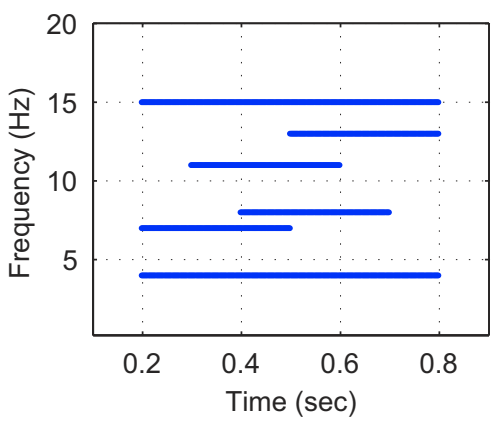

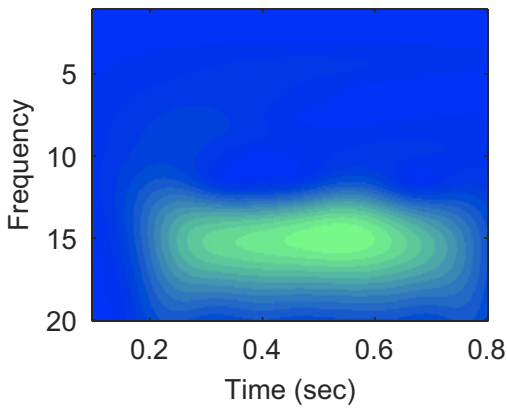

Fig. 12. The synthesized multicomponent aperiodic signal by the wavelet ridges method.

system is as shown in Fig. 3. In order to implement the circuit of the dynamical wavelet network some additions are made to the static wavelet network with Mexican Hat mother wavelet [17]. The dynamical wavelet network circuit is shown in Fig. 7a [18].
The capacitors are used as the memory elements which store the delayed versions of the output of the wavelet network. The voltage controlled switches are controlled by the external pulse generators which are triggered sequentially as shown in Fig. $7 \mathrm{~b}$ where the duration of the pulses satisfies 

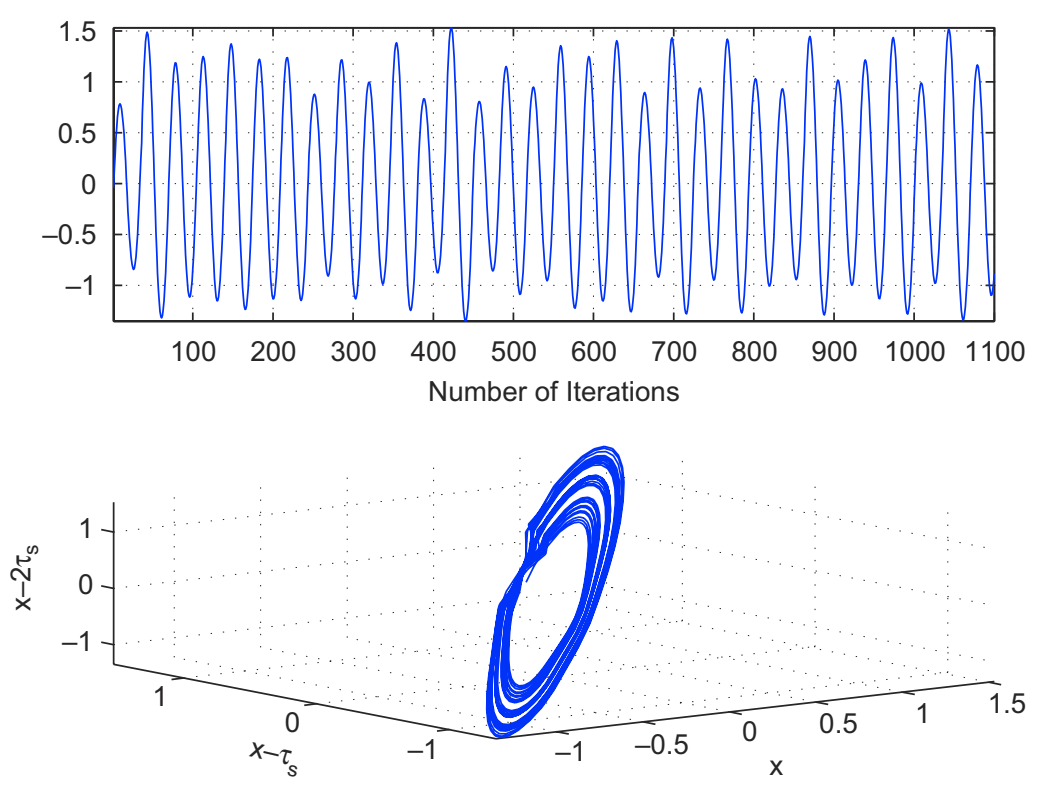

Fig. 13. The embedded multicomponent aperiodic signal in the phase space and the output of the wavelet network.

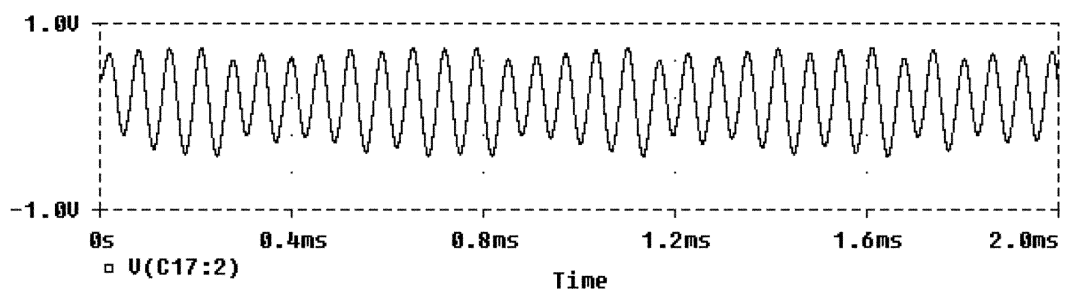

Fig. 14. The output of the circuit for multicomponent aperiodic signal.

$T_{d}<T$. The inputs of static wavelet network consists of the voltages on the capacitors.

\section{Verification}

In the proposed nonlinear circuit synthesis system the circuit has been designed according to the specifications given in the time-frequency domain as the wavelet ridges. When the design process has been completed, the output of the system must be tested to determine whether the proposed circuit satisfies the given conditions. Therefore, the wavelet ridges of the obtained signal should be determined. There are several ridge determination algorithms. The methods proposed in [22] for noisy monocomponent signals and in [23] for noisy multicomponent signals are successful in determination of the actual ridges. The proposed method based on the singular value decomposition (SVD) of the scalogram matrix [19] is computationally more faster than both methods. In the proposed method the singular value decomposition of the scalogram matrix of the related signal has been obtained. The effects of the additive white Gaussian noise (AWGN) is higher on the smaller singular values which correspond to the components of the signal with lower energy levels. Therefore, the effect of the noise is reduced by truncating the lower singular values. The approximated scalogram has been obtained by reconstructing the matrix by only using the larger singular values. Then the wavelet ridges are obtained by calculating the local maxima of the approximated scalogram matrix.

\section{Application}

The complete examples of the applications of the nonlinear circuit synthesis system will be given in this section. The synthesis of the circuit with monocomponent periodic output signal has been introduced in the first example. The second example includes the synthesis of the circuit with multicomponent aperiodic output signal.

\subsection{Example 1: monocomponent signal}

Signal synthesis program which uses the transform skeleton method has been written in MATLAB. When the time and frequency range is given, the pure sinusoidal signal, 


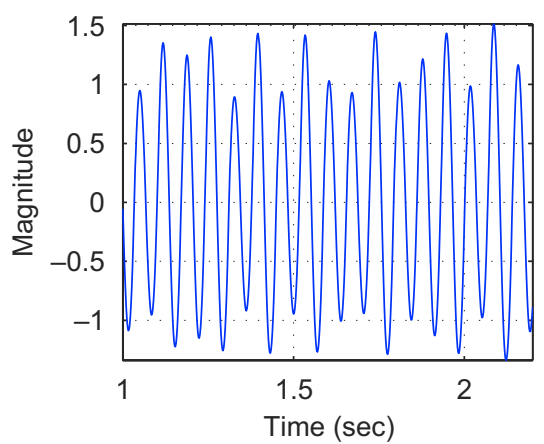

(a)

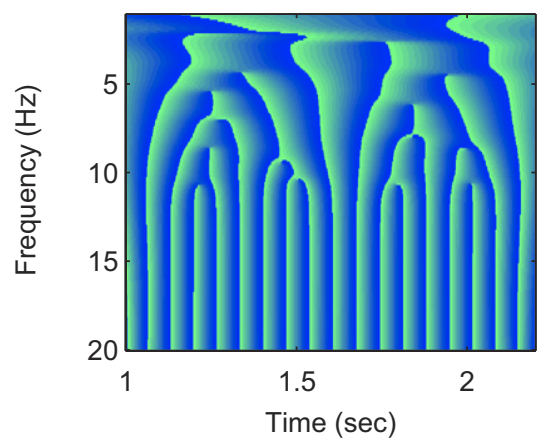

(c)

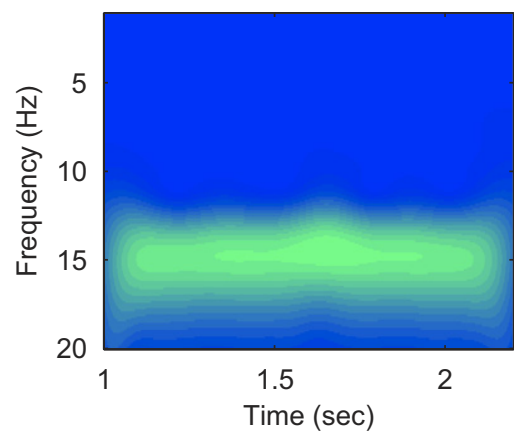

(b)

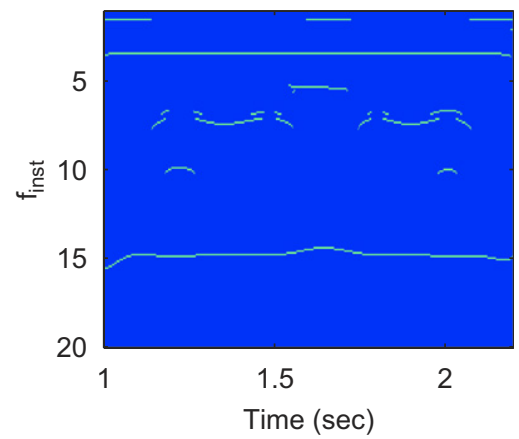

(d)

Fig. 15. (a) The time waveform, (b) the modulus, (c) the phase of the wavelet transform, and (d) the instantaneous frequency of the output of the wavelet network for multicomponent signal.

a linear or hyperbolic chirp can be synthesized. Also, the points in the ridge can be given interactively by the user and the ridges have been calculated by the linear interpolation. In order to illustrate the performance of the operation, the wavelet transform of the synthesized signal has been calculated.

The signal which have the energy distribution as

$$
E(t, f)= \begin{cases}1 & 1 \leqslant t<1.2 \mathrm{~s} \text { and } f=20 \mathrm{~Hz} \\ 0 & \text { otherwise }\end{cases}
$$

has been synthesized as shown in Fig. 8.

The time series obtained from the "Signal Synthesis" block is then transferred to the second block in order to determine the wavelet network parameters. The time series has been embedded by time-delay embedding by choosing the embedding dimension as $d_{\mathrm{e}}=3$ and the embedding delay as $T=\tau_{\mathrm{s}}$ where $\tau_{\mathrm{s}}$ is the sampling period and chosen as $\tau_{\mathrm{s}}=0.001$ in the signal synthesis process. The suitable wavelons have been selected by the stepwise selection by orthogonalization algorithm using the Mexican hat mother wavelet function. The number of wavelons has been selected as 3 and the algorithm has been implemented until the wavelet level 3. Then, the wavelet network has been trained by back-propagation training algorithm for 50 epochs. The time-delay embedded phase space and the output of the trained wavelet network are shown in Fig. 9.

After the determination of the wavelet network parameters, the wavelet network circuit has been designed by selecting the suitable wavelons and by adjusting the parameters of the wavelons and the amplifiers. The dynamical circuit structure has been given in Fig. 7. The output of the circuit in time-domain are shown in Fig. 10. The circuit is not sensitive to the initial conditions, i.e., the sinusoidal output signal has been reached at the steady state after short transients.

The wavelet ridges of the output of the wavelet network has been determined by SVD-based method proposed in [19]. The output of the wavelet network, the modulus and the phase of the wavelet transform and the instantaneous frequency of the signal are shown in Fig. 11.

\subsection{Example 2: multicomponent aperiodic signal}

The time-frequency domain specifications of the desired signal is as following

$$
E(t, f)= \begin{cases}0.2 & 0.2 \leqslant t<0.8 \mathrm{~s} \text { and } f=4 \mathrm{~Hz} \\ 0.3 & 0.2 \leqslant t<0.5 \mathrm{~s} \text { and } f=7 \mathrm{~Hz} \\ 0.2 & 0.4 \leqslant t<0.7 \mathrm{~s} \text { and } f=8 \mathrm{~Hz} \\ 0.1 & 0.3 \leqslant t<0.6 \mathrm{~s} \text { and } f=11 \mathrm{~Hz} \\ 0.2 & 0.5 \leqslant t<0.8 \mathrm{~s} \text { and } f=13 \mathrm{~Hz} \\ 1 & 0.2 \leqslant t<0.8 \mathrm{~s} \text { and } f=15 \mathrm{~Hz} \\ 0 & \text { otherwise }\end{cases}
$$

and the desired signal, target time-frequency plane, the synthesized signal and the wavelet transform of the synthesized signal are shown in Fig. 12. 
In order to train the wavelet network with Mexican hat mother wavelet function, the embedding delay has been chosen as $T=\tau_{\mathrm{s}}$ where the sampling period is $\tau_{\mathrm{s}}=0.002 \mathrm{~s}$ and the embedding dimension of $d_{\mathrm{e}}=3$. The number of wavelons in the wavelet network is 7 and the network has been trained for 1000 epochs. The obtained mean squared error is MSE $=0.000375857$. The output of the wavelet network in phase space and in time-domain are shown in Fig. 13.

The wavelet network circuit has been simulated in Spice. The output signal of the circuit in time domain are shown in Fig. 14.

The wavelet ridges of the output signal have been obtained by the SVD-based ridge determination algorithm. The results are shown in Fig. 15.

\section{Conclusions}

A nonlinear circuit synthesis method in wavelet domain has been proposed in this study. When the desired time-frequency domain specifications are given, the values of the parameters of the dynamical wavelet circuit have been determined in three steps. These are signal synthesis, the system modelling and the circuit synthesis. As a last step of the procedure, the accuracy of the synthesis has been investigated by the verification block.

\section{References}

[1] Cohen L. Time-frequency distributions - a review. Proc IEEE 1989;77:941-81.

[2] Chen P. Study of chaotic dynamical systems via time-frequency analysis. In: IEEE-SP international symposium on time-frequency and time-scale analysis, 1994. p. $357-60$.

[3] Lima E, Cohen L. Time-frequency analysis of harmonic oscillator motion. In: IEEE-SP international symposium on time-frequency and time-scale analysis, 1998. p. 25-8.

[4] Chandre C, Wiggins S, Uzer T. Time-frequency analysis of chaotic systems. Physica D 2003;181:171-96.

[5] Wong L, Chen JC. Nonlinear and chaotic behavior of structural system investigated by wavelet transform techniques. Int J Non-Linear Mech 2001;36:221-35.

[6] Özkurt N, Savacı F. Wavelet analysis of the generalized Chua's circuit. In: Proceedings of 9th workshop on nonlinear dynamics of electronic systems, The Netherlands, 2001. p. $100-4$.

[7] Galleani L, Biey M, Gilli M, Presti LL. Analysis of chaotic signals in the time-frequency plane. IEEE international conference on nonlinear signal and image processing NSIP99, Antalya, Turkey, 1999. p. 100-4.

[8] Özkurt N, Savacı F. Reconstruction of nonstationary signals along the wavelet ridges. Int J Bifurcation Chaos 2006;16: 191-8.

[9] Zhang Q, Benveniste A. Wavelet networks. IEEE Trans Neural Network 1992;3:889-98.

[10] Cao L, Hong Y, Fang H, He G. Predicting chaotic time series with wavelet networks. Physica D 1995;85:225-38.
[11] Allingham D, West M, Mees A. Wavelet reconstruction of nonlinear dynamics. Int J Bifurcation Chaos 1998;8: 2191-201.

[12] Oussar Y, Rivals I, Personnaz L, Dreyfus G. Training wavelet networks for nonlinear dynamic input-output modelling. Neurocomputing 1998;20:173-88.

[13] Zhang Q. Using wavelet network in nonparametric estimation. IEEE Trans Neural Network 1997;8:227-36.

[14] Özkurt N. Synthesis of nonlinear circuits in time-frequency domain. Ph.D. thesis, D.E.U. Graduate School of Natural and Applied Sciences, Turkey, 2004.

[15] Özkurt N, Savacı F. Signal synthesis using wavelet ridges. In: Proceedings of the IEEE 12th signal processing and communications applications conference, Kusadasi, Turkey, 2004. p. 489-92.

[16] Özkurt N, Savacı F, Gündüzalp M. The circuit implementation of a wavelet approximator. Int J Analog Integ Circ Signal Proc 2002;32:171-5.

[17] Özkurt N, Savacı F, Gündüzalp M. The circuit realization of Mexican hat wavelet function. AEU Int J Elect Commun 2005;59:370-3.

[18] Özkurt N, Savacı F. The implementation of nonlinear dynamical systems with wavelet network. AEU Int J Elect Commun 2005;60:338-44.

[19] Özkurt N, Savac1 F. Determination of wavelet ridges of nonstationary signals by singular value decomposition. IEEE Trans Circ Syst II: Express Briefs 2005;52:480-5.

[20] Mallat S. A wavelet tour of signal processing. San Diego, CA: Academic Press; 1999.

[21] Delprat N, Escudie B, Guillemain P, Kronland-Martinet R, Tchamitchian P, Torresani B. Asymptotic wavelet and Gabor analysis: extraction of instantaneous frequencies. IEEE Trans Inform Theory 1992;38:644-64.

[22] Carmona R, Hwang W. Characterization of signals by the ridges of their wavelet transforms. IEEE Trans Signal Proc 1997;45:2586-90.

[23] Carmona R, Hwang W, Torresani B. Multiridge detection and time-frequency reconstruction. IEEE Trans Signal Proc 1999;47:480-92.

[24] Abarbanel H. Analysis of observed chaotic data. New York: Springer; 1996.

[25] Pati Y, Krishnaprasad P. Analysis and synthesis of feedforward neural networks using discrete affine wavelet transformations. IEEE Trans Neural Network 1993;4: $73-85$.

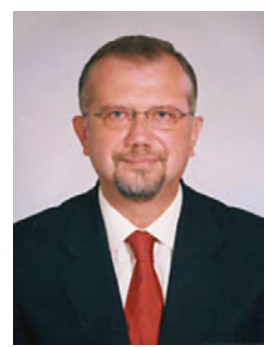

F. Acar Savac1 received B.S. degree in Electrical Engineering from the Middle East Technical University, Ankara, in 1979, the M.S. degree in Electrical Engineering from Bogaziçi University, Istanbul, in 1982 and Ph.D. degree in Electronics and Telecommunications from Istanbul Technical University, Istanbul, in 1989. He worked as postdoctoral fellow in Electronics Engineering Department of Katholieke

Universiteit, Leuven, Belgium in 1990-92. He worked at the Electrical-Electronics Engineering Faculty of Istanbul Technical 
University, Istanbul as a research assistant during1982-1990, as assistant professor in 1990-92 and associate professor since 1992 till 1998. He has been working at the Department of Electrical and Electronics Engineering in Izmir Institute of Technology, Izmir, since February 1998 as a full professor and as the chairman of the department. His research interests are linear and nonlinear system theory, chaos, artificial neural networks, fault analysis in analog circuits and signal processing.

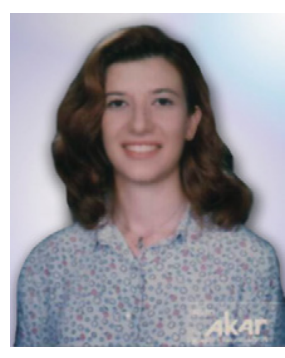

Nalan Özkurt received her B.S., M.S. and Ph.D. degree in Electrical and Electronics Engineering from the Dokuz Eylul University, in 1994, 1998 and 2004, respectively. She worked at Department of Electrical Engineering Dokuz Eylul University as a research assistant in 1995-2004 and she has been working at the same department as an Assistant Professor since 2004.

Her research interests are wavelets, nonlinear static and dynamical systems, chaos. She is a member of Association of Electrical and Electronic Engineers of Turkey. 